\title{
Erratum
}

\section{A bright early-type star in the halo of NGC 253: Runaway or in situ formation?}

\author{
F. Comerón ${ }^{1}$, A. E. Gómez ${ }^{2}$, and J. Torra ${ }^{3}$ \\ ${ }^{1}$ European Southern Observatory, Karl-Schwarzschild-Strasse 2, 85748 Garching bei Muenchen, Germany \\ e-mail: fcomeron@eso.org \\ 2 Observatoire de Paris-Meudon, DASGAL, UMR 8633 du CNRS, 92195 Meudon Cedex, France \\ e-mail: ana.gomez@obspm.fr \\ 3 Departament d'Astronomia i Meteorologia, Universitat de Barcelona, Av. Diagonal 647, 08028 Barcelona, Spain \\ e-mail: jordi@am.ub.es
}

A\&A, 400, 137-143 (2003), DOI: 10.1051/0004-6361:20021909

Key words. errata, addenda - galaxies: individual: NGC 253 - galaxies: starburst - stars: early-type - stars: formation - stars: kinematics

The coordinates of the object discussed in the paper "A bright early-type star in the halo of NGC 253: Runaway or in situ formation?" (A\&A, 400, 137) are in error. The actual coordinates are $\alpha(2000)=0^{\mathrm{h}} 48^{\mathrm{m}} 05.3^{\mathrm{s}}, \delta(2000)=-25^{\circ} 17^{\prime} 45^{\prime \prime}$. Accordingly, the correct denomination of the object following
IAU nomenclature is J004805.3-251745. The object is correctly identified in Fig. 2 of the paper.

The authors wish to express their gratitude to Dr. Ralf Scholz for kindly having pointed out the mistake to them. 